\title{
A term structure model under cyclical fluctuations in interest rates
}

Instituto

Complutense

de Análisis

Económico

\section{Manuel Moreno}

Department of Economic Analysis and Finance, University of Castilla-La Mancha, Toledo, Spain

Alfonso Novales

Instituto Complutense de Análisis Económico (ICAE), and

Department of Economic Analysis, Facultad de Ciencias Económicas y Empresariales, Universidad Complutense, Madrid, Spain

Federico Platania

Léonard de Vinci Pôle Universitaire,

Paris La Défense, France

\section{Abstract}

We propose a flexible yet tractable model of the term structure of interest rates (TSIR). Term structure models attempt to explain how interest rates depend on their maturities at a given point in time, characterizing the relationship between short-term and long-term rates. Our model can reproduce and fit a variety of TSIR shapes by capturing cyclical fluctuations of interest rates, different monetary policy reactions as witnessed pre- and post-crisis as well as the effect of the business cycle or exogenous shocks. Our modeling approach also provides a characterization of long-term fluctuations in the mean level of interest rates unveiling the effects of monetary policy interventions in interest rates. Furthermore, using daily US data, we compare the empirical ability of our model to both fit and forecast the TSIR under different economic scenarios. We show that our model improves pricing and risk management by fitting and predicting interest rates more accurately and precisely than do existing TSIR models.

Keywords Term structure of interest rates; cyclical fluctuations; bond pricing; TSIR fitting performance, interest rates forecast.

Jel Classification D53, E43, G13, C58, E32, C31.

\section{Working Paper no 1931 \\ September, 2019}

UNIVERSIDAD

COMPLUTENSE

MADRID 


\title{
A term structure model under cyclical fluctuations in interest rates
}

\begin{abstract}
We propose a flexible yet tractable model of the term structure of interest rates (TSIR). Term structure models attempt to explain how interest rates depend on their maturities at a given point in time, characterizing the relationship between short-term and long-term rates. Our model can reproduce and fit a variety of TSIR shapes by capturing cyclical fluctuations of interest rates, different monetary policy reactions as witnessed pre- and post-crisis as well as the effect of the business cycle or exogenous shocks. Our modelling approach also provides a characterization of long-term fluctuations in the mean level of interest rates unveiling the effects of monetary policy interventions in interest rates. Furthermore, using daily US data, we compare the empirical ability of our model to both fit and forecast the TSIR under different economic scenarios. We show that our model improves pricing and risk management by fitting and predicting interest rates more accurately and precisely than do existing TSIR models.
\end{abstract}

Keywords: Term structure of interest rates; cyclical fluctuations; bond pricing; TSIR fitting performance, interest rates forecast.

JEL classification: D53, E43, G13, C58, E32, C31. 


\section{Introduction}

Zero-coupon interest rates at very short maturities are determined by the implementation of monetary policy. However, longer term zero-coupon interest rates are the drivers relevant for private sector decisions since the cost of the credit needed to finance durable consumption purchases and investment goods is usually related to the interest rates at longer maturities, such as 10 -year rates. The reference to zero-coupon rates reflects the fact that these specify the required payment on the return of an amount of money loaned over a given period of time, the maturity of the interest rate. The price of a bond or any other financial asset involving a flow of payments can be easily determined using zero-coupon rates at the maturities at which a given flow will take place in the future.

The so-called term structure of interest rates (TSIR) is the representation of zero-coupon interest rates as a function of the time to maturity. It codifies the relationship between short-term rates and medium-term and long-term rates. The TSIR is an essential input for the analysis of fixed-income markets as well as for monetary policy design and implementation, and, over the years, many models have been proposed to describe the nature of the relationships between zero-coupon interest rates at different maturities. This amounts to estimating how monetary policy interventions affect the costs of consumption and investment decisions and how they potentially influence the business cycle in a given economy.

TSIR models can be endogenous or exogenous. Endogenous models consider changes in interest rates at different maturities as being determined by one or more common factors for which they assume a specific stochastic behavior. Under such assumptions the current TSIR can be derived from the model. See, for instance, Vasicek (1977), Cox et al. (1985), Duffie and Kan (1996), Moreno and Platania (2015), or Renne (2016), among others.

In contrast, exogenous models consider the current TSIR as an input and derive the future changes in interest rates that prevent intertemporal arbitrage opportunities, i.e. profitable trading strategies that could be implemented by combining available fixed income assets with different maturities. Some examples are Hull and White (1990, 1993) and Heath et al. (1992). For detailed reviews on TSIR models see James and Webber (2001), Brigo and Mercurio (2006), Nawalkha et al. (2007), Filipović (2009), Andersen and Piterbarg (2010), and Munk (2015), among others.

We work in a standard framework of one-factor continuous-time endoge- 
nous models for the TSIR with the instantaneous spot rate as its single factor. In particular, we regard the instantaneous zero-coupon interest rate as following an Ornstein-Uhlenbeck stochastic process. Consequently, its time evolution is governed by the tendency to revert to the long-term mean while reacting to shocks from a fundamental Wiener process describing interest rate innovations.

What is new in our proposal is the assumption that the long-term mean of the instantaneous zero-coupon interest rate displays cyclical behavior. This cyclical behavior is modelled through a Fourier series. This specification allows us to simulate the wide variety of shapes actually observed for the TSIR. The cyclical behavior in the long-term mean may arise because of reactions to monetary policy interventions intended to influence the business cycle.

Indeed, the relationship between the term structure and macroeconomic variables has been widely documented in the literature (see Diebold et al. (2006), Evans and Marshall (2007), Tillmann (2007), and Paccagnini (2016), among many others). Our specification is in the spirit of the Hull and White (1994) term structure model in that it considers time varying parameters. We go one step further in dealing with seasonality explicitly as well as in allowing for a specific dependence of interest rates on the business cycle. Even though we use it in this paper to estimate the daily term structure of interest rates, our modelling strategy could also be used to estimate the time variation in the unobserved mean level in interest rates. Among other things, that would allow for evaluating the relevance of monetary policy in interest rate determination, as it is done in Mallik et al. (2017).

Our proposal is an alternative to models usually adopted by central banks, such as Nelson and Siegel (1987) and Svensson (1994), that achieve this kind of flexibility by using exponential functions while obtaining a good fit to market interest rates. In these models, the instantaneous interest rate expected at any future time is described by a combination of exponential functions of the given time horizon. Its use as the single factor of the TSIR means that interest rates at any other maturity are also expressed by combinations of exponential functions of their maturities.

Similarly, the Ornstein-Uhlenbeck specification for the instantaneous rate means that any other interest rate is expressed as a nonlinear function of its maturity. Moreover, in our model, interest rates follow a Gaussian distri- 
bution, ${ }^{1}$ a relevant issue given the theoretical zero bound on interest rates and the negative interest rates that have been observed recently in some countries. $^{2}$

Estimating the parameters in the representations for interest rates at the different maturities we can recover values for parameters in the process for the instantaneous interest rate as well as for parameters in the Fourier series describing the cyclical behavior of the mean reversion level. We analyze the in-sample and out-of-sample performance of this model to explain the TSIR, using Vasicek (1977) and Nelson and Siegel (1987) as benchmark models.

Vasicek (1977) was one of the first TSIR models and it has inspired a number of interesting extensions. Its main assumptions are that interest rates converge to a (constant) long-term mean and that the volatility of changes in interest rates is constant. Consequently, interest rates follow a Gaussian distribution. As our model generalizes Vasicek (1977), the Vasicek model is a natural benchmark with which to assess the gains attained by our modelling of the mean level of interest rates and the relevance of its (potential) flexibility.

On the other hand, the Nelson and Siegel (1987) model is a significant benchmark since it is one of the most popular models of the yield curve and is used by many central banks in the implementation and evaluation of monetary policy. Moreover, Diebold and Li (2006) showed the good forecasting performance of this model in comparison with ten alternative competitors, including Fama and Bliss (1987) and Cochrane and Piazzesi (2005).

With respect to our data set, our model outperforms its competitors both in-sample and out-of-sample. It provides a more precise fit to actual market values as well as better forecasts.

The paper is organized as follows. Section 2 introduces the analytical model and characterizes the TSIR. Section 3 presents the in-sample and outof-sample empirical analyses of the model. Finally, Section 4 summarizes the main findings and conclusions.

\footnotetext{
${ }^{1}$ In usual market conditions such a distribution is considered a disadvantage, and this has often motivated the use of CIR-type models, which impose the positivity of interest rates. We strongly thank one of the referees for focusing our attention on this issue.

${ }^{2}$ For a deep analysis of Gaussian TSIR models see Realdon (2016) and references therein.
} 


\section{The Term Structure Model}

In this section we introduce the model, present the partial differential equation that must be satisfied by the price of any derivative asset, obtain bond pricing equations, and characterize the TSIR.

\subsection{The Model}

Let $r_{t}$ denote the instantaneous interest rate at time $t$. We assume that the time evolution of $r_{t}$ is given by an Ornstein-Uhlenbeck process, defined by the stochastic differential equation

$$
d r_{t}=\kappa\left(f(t)-r_{t}\right) d t+\sigma d W_{t}
$$

where $\kappa, \sigma \in \mathbb{R}^{+}$and $W_{t}$ is a standard Wiener process. In addition, we assume that the mean-reversion level, $f(t)$, follows a time-dependent process driven by a Fourier series,

$$
f(t)=\sum_{n=0}^{\infty} R e\left[A_{n} e^{i n \omega t}\right],
$$

where we only consider the real part of the Fourier series since it is the only part that makes economic sense. Note that $A_{n} \in \mathbb{C}$ for all $n$, so there is a phase factor contained in $A_{n}$. In more detail, $A_{n}=A_{n, x}+i A_{n, y}$ where $A_{n, x}, A_{n, y} \in \mathbb{R}$. Hence, $A_{n, x}$ and $A_{n, y}$ denote, respectively, the amplitude and phase of the fluctuations of the instantaneous rate. Finally, this model specializes to that in Vasicek (1977) by taking $A_{n}=0$ for $n \in \mathbb{N}-\{0\}$.

Now, let $\Lambda\left(r_{t}, t\right)$ denote the market price of risk, which is assumed to be constant, $\Lambda\left(r_{t}, t\right)=\lambda$. Then the risk-neutral version of the process (1) is given by

$$
d r_{t}=\mu_{r} d t+\sigma d \widetilde{W}_{t}
$$

for

$$
\begin{aligned}
\mu_{r} & =\kappa\left(\alpha+g(t)-r_{t}\right), \\
\alpha & =A_{0}-\frac{\lambda \sigma}{\kappa}, \\
g(t) & =\sum_{n=1}^{\infty} \operatorname{Re}\left[A_{n} e^{i n \omega t}\right]=f(t)-A_{0},
\end{aligned}
$$


where $A_{0} \in \mathbb{R}$ and $\widetilde{W}_{t}=W_{t}+\lambda t$ is a standard Wiener process under the risk-neutral measure $\widetilde{P}$. The following proposition describes the solution of the stochastic differential equation (2).

Proposition 2.1. The solution of the risk-neutral process followed by the instantaneous interest rate is given by ${ }^{3}$

$$
\begin{aligned}
r_{s} & =e^{-\kappa(s-t)} r_{t}+\left(1-e^{-\kappa(s-t)}\right) \alpha \\
& +\sum_{n=1}^{\infty} \operatorname{Re}\left[\frac{\kappa A_{n}}{\kappa+i n \omega}\left(e^{i n \omega s}-e^{-\kappa(s-t)+i n \omega t}\right)\right]+\sigma \int_{t}^{s} e^{-\kappa(s-u)} d \widetilde{W}_{u} .
\end{aligned}
$$

From Proposition 2.1, it is clear that the instantaneous interest rate follows a normal distribution. Its first two statistical moments under $\widetilde{P}$ are given by

$$
\begin{aligned}
\widetilde{E}\left[r_{T} \mid r_{t}\right]= & e^{-\kappa(T-t)} r_{t}+\left(1-e^{-\kappa(T-t)}\right) \alpha \\
& +\sum_{n=1}^{\infty} R e\left[\frac{\kappa A_{n}}{\kappa+i n \omega}\left(e^{i n \omega T}-e^{-\kappa(T-t)+i n \omega t}\right)\right], \\
\widetilde{V}\left[r_{T} \mid r_{t}\right]= & \widetilde{V}\left[\sigma \int_{t}^{T} e^{-\kappa(T-u)} d \widetilde{W}_{u}\right]=\left(\sigma \int_{t}^{T} e^{-\kappa(T-u)} d \widetilde{W}_{u}\right)^{2} \\
= & \sigma^{2} \int_{t}^{T} e^{-2 \kappa(T-u)} d u=\frac{\sigma^{2}}{2 \kappa}\left(1-e^{-2 \kappa(T-t)}\right),
\end{aligned}
$$

where, in calculating the variance, we have applied the isometry property of the stochastic integral.

\subsection{Pricing and the TSIR}

Let $P\left(r_{t}, t, T\right)$ denote the price at time $t$ of a zero-coupon bond that pays $\$ 1$ at maturity $T$. Applying the Itô Lemma, standard no arbitrage arguments and some trivial algebra, we get the partial differential equation (PDE)

$$
\frac{1}{2} \sigma_{r}^{2} P_{r r}\left(r_{t}, t, T\right)+\left(\mu_{r}-\Lambda\left(r_{t}, t\right) \sigma_{r}\right) P_{r}\left(r_{t}, t, T\right)+P_{t}\left(r_{t}, t, T\right)-r_{t} P\left(r_{t}, t, T\right)=0,
$$

\footnotetext{
${ }^{3}$ This result follows from the observation that, as $e^{-\kappa(s-t)}$ is square-integrable in $[t, s]$, it belongs to a Hilbert space.
} 
that must be satisfied by the price of any derivative.

Substituting the expression (1) and the constant market price of risk, $\lambda$, into (5), we get the PDE for the bond price,

$$
\frac{1}{2} \sigma^{2} P_{r r}+\kappa\left(\alpha+g(t)-r_{t}\right) P_{r}+P_{t}-r_{t} P=0
$$

subject to the terminal condition $P\left(r_{T}, T, T\right)=1$ for all $r_{T}$.

Using probabilistic techniques, the solution of this PDE can be written as a risk-neutral conditional expectation, that is,

$$
P\left(r_{t}, t, T\right)=\widetilde{E}\left[e^{-\int_{t}^{T} r_{s} d s} \mid r_{t}\right]
$$

From Proposition 2.1 it is clear that $\int_{t}^{T} r_{s} d s$ is a random normal variable. Straightforward algebra leads to the solution of this PDE given in the following proposition.

Proposition 2.2. The price at time $t$ of a zero-coupon bond with maturity $T$ and face value $\$ 1$ is given by

$$
P\left(r_{t}, t, T\right)=\exp \left(-\widetilde{E}\left[\int_{t}^{T} r_{s} d s \mid r_{t}\right]+\frac{1}{2} \widetilde{V}\left[\int_{t}^{T} r_{s} d s \mid r_{t}\right]\right)
$$

where

$$
\begin{aligned}
\widetilde{E}\left[\int_{t}^{T} r_{s} d s \mid r_{t}\right] & =\frac{1-e^{-\kappa(T-t)}}{\kappa} r_{t}-\left(\frac{1-e^{-\kappa(T-t)}}{\kappa}-(T-t)\right) \alpha \\
& +\sum_{n=1}^{\infty} \operatorname{Re}\left[\frac{e^{i n \omega t}\left(n \omega e^{-\kappa(T-t)}+i \kappa-n \omega\right)-i \kappa e^{i n \omega T}}{n \omega(\kappa+i n \omega)} A_{n}\right] \\
\tilde{V}\left[\int_{t}^{T} r_{s} d s \mid r_{t}\right] & =\frac{\sigma^{2}}{\kappa^{2}}\left[(T-t)-2 \frac{1-e^{-\kappa(T-t)}}{\kappa}+\frac{1-e^{-2 \kappa(T-t)}}{2 \kappa}\right]
\end{aligned}
$$

Since all affine models yield an exponential-affine functional form for bond pricing, we can immediately rewrite the previous proposition to obtain the next one. 
Proposition 2.3. The price at time $t$ of a zero-coupon bond with maturity $T$ and $\$ 1$ face value is given by

$$
P\left(r_{t}, t, T\right)=e^{A(t, T)-B(t, T) r_{t}},
$$

where

$$
\begin{aligned}
A(t, T) & =\frac{\sigma^{2}}{2 \kappa^{2}}\left[(T-t)-2 B(t, T)+\frac{1-e^{-2 \kappa(T-t)}}{2 \kappa}\right]+(B(t, T)-(T-t)) \alpha \\
& -\sum_{n=1}^{\infty} \operatorname{Re}\left[\frac{A_{n}}{n \omega(\kappa+i n \omega)}\left(e^{i n \omega t}\left(n \omega e^{-\kappa(T-t)}+i \kappa-n \omega\right)-i \kappa e^{i n \omega T}\right)\right] \\
B(t, T) & =\frac{1-e^{-\kappa(T-t)}}{\kappa} .
\end{aligned}
$$

The following corollary is immediate.

Corollary 2.4. As a coupon bond can be interpreted as a portfolio of zerocoupon bonds, the price of a coupon bond can be obtained applying Proposition 2.2 or 2.3.

With this framework and the bond price $P\left(r_{t}, t, T\right)$ given by Proposition 2.3 , the TSIR is fully characterized in the following corollary.

Corollary 2.5. The yield to maturity, $R\left(r_{t}, t, T\right)$, is given by

$$
R\left(r_{t}, t, T\right)=-\frac{1}{\tau} \ln \left(P\left(r_{t}, t, T\right)\right), \tau=T-t
$$

The short-term interest rate is defined as the instantaneous interest rate at time $t$, that is,

$$
r_{t}=\lim _{\tau \rightarrow 0} R\left(r_{t}, t, T\right)=R\left(r_{t}, t, t\right) .
$$

The instantaneous forward rate is given as

$$
f\left(r_{t}, t, T\right)=-\frac{\partial \ln \left(P\left(r_{t}, t, T\right)\right)}{\partial T} .
$$


Figure 1 illustrates the flexibility of our model, which can give rise to different yield curves having different shapes: upward sloping, downward sloping, humped, and inverted humped. For comparison, we also show the yield curve coming from the Vasicek model. Exponential representations as in the Nelson-Siegel and Svensson TSIR models were proposed because they serve to reproduce the changes from concavity to convexity that are sometimes observed in empirical yield curves. ${ }^{4}$

Furthermore, our model is highly tractable analytically for fixed income option pricing. For instance, consider a call option maturing at time $T_{c}$ with strike $K$, written on a zero-coupon bond that matures at time $T_{b}>T_{c}$. Let $c_{t}\left(r_{t}, T_{c}, T_{b}, K\right)$ denote the price of this call option at time $t$. Then the boundary condition of the PDE (6) is given by

$$
c_{T_{c}}\left(r_{T_{c}}, T_{c}, T_{b}, K\right)=\max \left\{P\left(r_{T_{c}}, T_{c}, T_{b}\right)-K, 0\right\}
$$

Hence, under the risk-neutral measure $\widetilde{P}$, the price of this option at time $t$ is given by

$$
c_{t}\left(r_{t}, T_{c}, T_{b}, K\right)=\widetilde{E}\left[e^{-\int_{t}^{T_{c}} r_{s} d s}\left(P\left(r_{T_{c}}, T_{c}, T_{b}\right)-K\right)^{+} \mid r_{t}\right] .
$$

The call option price is given by the following proposition.

Proposition 2.6. The price at time $t$ of a European call option with maturity $T_{c}$ written on a zero-coupon bond expiring at time $T_{b}$ and face value $\$ 1$ is given by

$$
\begin{aligned}
c_{t}\left(r_{t}, T_{c}, T_{b}, K\right) & =P\left(r_{t}, t, T_{c}\right) \widetilde{E}\left[\left(P\left(w_{T_{c}}, T_{c}, T_{b}\right)-K\right)^{+} \mid r_{t}\right] \\
& =P\left(r_{t}, t, T_{c}\right) F\left(r_{t}, t, T_{c}, T_{b}\right) \Phi\left(d_{1}\right)-P\left(r_{t}, t, T_{c}\right) K, \Phi\left(d_{2}\right),
\end{aligned}
$$

where the bond price $P\left(r_{t}, t, \cdot\right)$ is given by Proposition 2.2, $\Phi(\cdot)$ denotes the standard normal distribution, $F\left(r_{t}, t, T_{c}, T_{b}\right)$ represents the value at time $t$ of

\footnotetext{
${ }^{4}$ The working paper [Moreno et al. (2016)] includes graphs showing the term structure of zero-coupon bond prices as well as the TSIR of yields under the Vasicek and Fourier models for different values of the parameters $\kappa, \sigma$, and $\alpha$. It also shows how the TSIR in the Fourier model responds to changes in the parameters $A_{n, x}, A_{n, y}$, and $\omega$. All these representations illustrate that our proposed Fourier model provides great flexibility even when only a small number of terms in the Fourier expansion are used.
} 
a forward contract bond maturing at time $T_{c}$ written on a zero-coupon bond with maturity date $T_{b}$, and

$$
\begin{aligned}
d_{1} & =\frac{\ln \left(\frac{F\left(r_{t}, t, T_{c}, T_{b}\right)}{K}\right)+\frac{1}{2} B\left(T_{c}, T_{b}\right)^{2} \widetilde{V}\left[w_{T_{c}} \mid r_{t}\right]}{B\left(T_{c}, T_{b}\right) \sqrt{\widetilde{V}\left[w_{T_{c}} \mid r_{t}\right]}}, \\
d_{2} & =d_{1}-B\left(T_{c}, T_{b}\right) \sqrt{\widetilde{V}\left[w_{T_{c}} \mid r_{t}\right]},
\end{aligned}
$$

with $B\left(T_{c}, T_{b}\right)$ as in (8) and $\widetilde{V}\left[w_{T_{c}} \mid r_{t}\right]=\widetilde{V}\left[r_{T} \mid r_{t}\right]$ as in (4).

\section{Empirical Analysis}

In this section we compare the empirical performance of our Fourier model with two benchmarks, Vasicek (1977) and Nelson and Siegel (1987). Since we have analytical expressions for interest rates and bond prices, we could use realized data on either of these variables to evaluate the model. We decided to use interest rate data, even though a good fit to yields does not guarantee a good fit to bond prices due to the nonlinearity of the mapping from yields to prices. Analyzing the empirical performance of our model for bond prices could be an interesting issue for further research.

To keep the specification of our model simple, we will consider only the first $(n=1)$ term in the Fourier series in (3). As mentioned before, the Vasicek specification is a special case of our model, while Nelson and Siegel (1987) suggested to model the yield curve at a point in time by

$$
R(\tau)=\beta_{1}+\beta_{2} \frac{1-\exp (-\lambda \tau)}{\lambda \tau}+\beta_{3}\left(\frac{1-\exp (-\lambda \tau)}{\lambda \tau}-\exp (-\lambda \tau)\right)
$$

where $\tau$ is the time to maturity, and $\beta_{1}, \beta_{2}, \beta_{3}$, and $\lambda$ are constant parameters. The parameters $\beta_{1}, \beta_{2}$, and $\beta_{3}$ are closely related to the level, slope, and

curvature of the yield curve, respectively, while $\lambda$ governs the exponential decay rate.

\subsection{Data}

The data set used for the empirical study consists of daily US Treasury yield curve rates. In more detail, we take daily observations of the Treasury constant maturity interest rates for $1,3,6,12,24,36,60,84,120,240$, and 
360 months, from July 31, 2001, to June 20, 2016. The 30-year Treasury constant maturity series was discontinued on February 18, 2002, and reintroduced on February 9, 2006. During this period, the US Treasury published an adjustment factor that can be used for estimation of the 30-year nominal rate from the observed daily nominal 20-year interest rate.

In the temporally late part of the sample, short rates took very low values, being equal to zero at some points. We will therefore use absolute measures when evaluating forecasts since at such interest rate levels, relative measures of forecast errors might be huge even for small absolute errors.

\subsection{In-sample model fitting}

We start by assessing the in-sample fitting of the three models using the whole sample period, with 3,724 daily data points for interest rates at each maturity. As in Diebold and Li (2006), we estimate the parameters at each point in time $t$ to obtain a time series of parameter estimates. Diebold and Li estimated the linear version of the Nelson-Siegel function that arises upon fixing the numerical value of the decay parameter $\lambda$, whereas we solve a nonlinear estimation problem for each day in the sample to obtain daily estimates for the structural parameters $\theta_{t}^{N S}=\left(\beta_{1, t}, \beta_{2, t}, \beta_{3, t}, \lambda_{t}\right)$.

To approximate our proposed model, we plug the expression for the zerocoupon bond price $P\left(r_{t}, t, T\right)$ from Proposition 2.3 into Corollary 2.5. This allows us to formulate the problem of minimizing the sum of squared errors in the form of a regression model. Indeed, for each maturity $j$, taking just the first term in (3), the structural parameters of the yield curve for the Fourier model can be estimated from

$$
Y_{j, t}=\sum_{i=1}^{4} \delta_{i, t} z_{i j, t}+u_{j, t},
$$


where

$$
\begin{aligned}
Y_{j, t} & =R\left(r_{t}, t, T_{j}\right)-\frac{B\left(t, T_{j}\right)}{T_{j}-t} r_{t}, \\
\left\{\delta_{i, t}\right\}_{i=1}^{4} & =\left\{\alpha_{t}, \sigma_{t}^{2}, A_{x, t}, A_{y, t}\right\} \\
z_{1 j, t} & =\frac{B\left(t, T_{j}\right)}{T_{j}-t}-1, \\
z_{2 j, t} & =\frac{1}{2 \kappa_{t}^{2}}-\frac{B\left(t, T_{j}\right)}{\left(T_{j}-t\right) \kappa_{t}^{2}}+\frac{1-e^{-2 \kappa_{t}\left(T_{j}-t\right)}}{4\left(T_{j}-t\right) \kappa_{t}^{3}}, \\
B\left(t, T_{j}\right) & =\frac{1-e^{-\kappa_{t}\left(T_{j}-t\right)}}{\kappa_{t}},
\end{aligned}
$$

while the term $R e\left[\frac{A_{x, t}+i A_{y, t}}{\omega_{t}\left(\kappa_{t}+i \omega_{t}\right)}\left(e^{i \omega_{t} t}\left(\omega_{t} e^{-\kappa_{t}\left(T_{j}-t\right)}+i \kappa_{t}-\omega_{t}\right)-i \kappa_{t} e^{i \omega_{t} T_{j}}\right)\right]$ that appears in the expression for the yield to maturity $R\left(r_{t}, t, T_{j}\right)$, becomes $\delta_{3, t} z_{3 t}+\delta_{4, t} z_{4 t}$.

The error term $u_{j, t}$ in (9) can be interpreted as the approximation error in the practical implementation of the yield model resulting from considering only one term of the infinite Fourier expansion. The difficulty with this model is that the explanatory variables are functions of the structural parameters $\kappa_{t}$ and $\omega_{t}$, so we cannot estimate it via a simple regression involving observable variables. Our strategy is to use again the cross-section of interest rates observed each day in the sample to solve a nonlinear optimization problem for the values of $\kappa_{t}, \omega_{t}$, and $\left\{\delta_{i, t}\right\}_{i=1}^{4}$ that minimizes the sum of squared residuals in (9), $S R\left(\hat{\theta}_{t}\right)=\sum_{j, t}\left(Y_{j, t}-\sum_{i=1}^{4} \hat{\delta}_{i, t} \hat{z}_{i j, t}\right)^{2}$. After estimation, we can recover the values of the structural parameters $\theta_{t}^{F}=\left(\alpha_{t}, \sigma_{t}^{2}, A_{x, t}, A_{y, t}, \kappa_{t}, \omega_{t}\right)$. As a result, we obtain time series for each parameter in $\theta_{t}^{F}$ over the sample period.

The Vasicek model arises upon setting $z_{3 t}=z_{4 t}=0$ in (9). In this case, we use a similar procedure to estimate the structural parameters $\theta_{t}^{V}=$ $\left(\alpha_{t}, \sigma_{t}^{2}, \kappa_{t}\right)$.

To estimate $\delta_{1, t}$ and $\beta_{1, t}$ we used the daily levels of the long-term 30year rate as the initial conditions. For the remaining parameters, we used as the initial conditions on each day the estimates obtained for the previous day. To avoid potential instability in parameter estimates, we imposed an upper bound on daily changes. Table 1 shows the means and standard deviations of the daily estimates for the parameters of the three models over the whole sample. The last two rows show the minimized numerical values of 
the objective function, $\operatorname{argmin}_{\theta_{t}} S R\left(\theta_{t}\right)$, and the sum of the absolute fitting errors, $\sum_{i, t}\left|\hat{u}_{i, t}\right|$, over the whole sample and across all maturities. These are measures of the fit of each model to the observed daily yield curves.

For the observed yield curve, the Fourier model fits the in-sample significantly better than do the alternative benchmarks. It reduces the aggregate sum of squares by $23 \%$ relative to the Nelson Siegel model. The Fourier and Nelson Siegel models reduce the sum of squares by $79 \%$ and $84 \%$ with respect to the the Vasicek benchmark. ${ }^{5}$ This is an interesting result since we are estimating the Fourier model with $n=1$. It is encouraging to know that we do not need to use more terms of the Fourier series to achieve a good representation of the TSIR. ${ }^{6}$ Increasing the number of terms in the Fourier expansion would eventually allow for fitting the observed yield curve arbitrarily well.

Table 2 disaggregates fitting statistics over the TSIR, excluding the shortterm rate. Over the whole sample period, the Fourier and the Nelson-Siegel models perform similarly, as both attain the lowest residual sum of squares over five maturities. The simpler Vasicek model does not beat the two competitor models for any maturity.

\subsection{Out-of-sample forecasting}

Having compared the in-sample fit of the three models of the yield curve, we now analyze their forecasting performance 1, 5, and 21 days ahead. In the Nelson-Siegel model, interest rate forecasts at different maturities are readily obtained once we get forecasts for the parameter values, as shown below. The explanatory variables in the Vasicek and Fourier models are functions of the time to maturity and the parameters, so that we can obtain their expected future values conditional on a given forecasting model for the parameters. In these two models, the instantaneous interest rate appears as a right-hand side variable. So, we start by obtaining the forecast for the instantaneous interest rate from the Euler discretization $E\left[r_{t+\Delta t} \mid r_{t}\right]=r_{t}+\kappa\left(\mu-r_{t}\right) \Delta t$, where $\Delta t$ is the forecast horizon in days and $\mu$ is a single parameter in the

\footnotetext{
${ }^{5}$ The Fourier model reduces the sum of absolute values of fitting errors over the whole sample by $13 \%$ relative to the Nelson Siegel model. The Fourier and Nelson Siegel models reduce this statistic by $58 \%$ and $63 \%$ relative to the Vasicek benchmark.

${ }^{6}$ Imposing upper bounds on daily parameter changes constitutes a serious disadvantage for the Fourier model, which, were no restrictions imposed, would deliver an aggregate sum of squares error of 0.0055 with an improvement of $74 \%$ relative to the Nelson-Siegel model.
} 
Vasicek model and it is a nonlinear function of the structural parameters in the Fourier model. Once we have this forecast, we can easily compute the forecasted yield rate at each maturity. For the Fourier model we maintain the simplest choice of a single term $(n=1)$ in the Fourier series when computing out-of-sample forecasts.

To compute forecasts for parameter values, at each point in time $t$ we estimate a first-order vector autoregression for the time series of estimated parameters up to that period, $\hat{\theta}_{t+h}$. Such parameter dynamics are justified in the literature (see Diebold and Li (2006) and Gourieroux and Monfort (2013), among others). Over the whole sample, residuals from this model seem to be autocorrelation free. Then, we obtain the predicted values for the parameters, $\tilde{\theta}_{t+h}$, for the required forecast horizon as a function of the estimated values using information up to time $t .^{7}$. We use the following specifications for the models.

1. Vasicek model:

$$
\left(\begin{array}{c}
\tilde{\alpha}_{t+h} \\
\tilde{\sigma}_{t+h} \\
\tilde{\kappa}_{t+h}
\end{array}\right)=\left(\begin{array}{c}
\hat{c}_{1} \\
\hat{c}_{2} \\
\hat{c}_{3}
\end{array}\right)+\left(\begin{array}{ccc}
\hat{\gamma}_{1} & 0 & 0 \\
0 & \hat{\gamma}_{2} & 0 \\
0 & 0 & \hat{\gamma}_{3}
\end{array}\right) \times\left(\begin{array}{c}
\hat{\alpha}_{t+h-1} \\
\hat{\sigma}_{t+h-1} \\
\hat{\kappa}_{t+h-1}
\end{array}\right) .
$$

The predicted value of the yield to maturity is given by

$$
\tilde{R}\left(r_{t+h}, t+h, T_{j}\right)=\left(z_{1 j, t+h}+1\right) r_{t+h}+z_{1 j, t+h} \tilde{\alpha}_{t+h}+z_{2 j, t+h} \tilde{\sigma}_{t+h}^{2} .
$$

2. Nelson-Siegel model:

$$
\left(\begin{array}{c}
\tilde{\beta}_{1, t+h} \\
\tilde{\beta}_{2, t+h} \\
\tilde{\beta}_{3, t+h} \\
\tilde{\lambda}_{t+h}
\end{array}\right)=\left(\begin{array}{c}
\hat{c}_{1} \\
\hat{c}_{2} \\
\hat{c}_{3} \\
\hat{c}_{4}
\end{array}\right)+\left(\begin{array}{cccc}
\hat{\gamma}_{1} & 0 & 0 & 0 \\
0 & \hat{\gamma}_{2} & 0 & 0 \\
0 & 0 & \hat{\gamma}_{3} & 0 \\
0 & 0 & 0 & \hat{\gamma}_{4}
\end{array}\right) \times\left(\begin{array}{c}
\hat{\beta}_{1, t+h-1} \\
\hat{\beta}_{2, t+h-1} \\
\hat{\beta}_{3, t+h-1} \\
\hat{\lambda}_{t+h-1}
\end{array}\right) .
$$

\footnotetext{
${ }^{7}$ VAR forecasts at the different horizons were computed using the varf.m Matlab routine in the spatial econometrics library by Prof. James LeSage, available at http://www.spatial-econometrics.com/

The routine considers a model specification $\tilde{y}_{t+1}=\hat{c}+\hat{\gamma} \cdot \hat{y}_{t}$ and, to obtain forecasts $h$-periods ahead ( $h=1,5,21$ days), it loops over the equation $h$-times.
} 
The predicted value of the yield to maturity is given by

$$
\begin{gathered}
\tilde{R}\left(r_{t+h}, t+h, T_{j}\right)=\tilde{\beta}_{1+h}+\tilde{\beta}_{2 t+h} \frac{1-\exp \left(-\tilde{\lambda}_{t+h}\left(T_{j}-(t+h)\right)\right)}{\tilde{\lambda}_{t+h}\left(T_{j}-(t+h)\right)} \\
+\tilde{\beta}_{3 t+h}\left(\frac{1-\exp \left(-\tilde{\lambda}_{t+h}\left(T_{j}-(t+h)\right)\right)}{\tilde{\lambda}_{t+h}\left(T_{j}-(t+h)\right)}-\exp \left(-\tilde{\lambda}_{t+h}\left(T_{j}-(t+h)\right)\right)\right) .
\end{gathered}
$$

3. Fourier model:

$$
\left(\begin{array}{c}
\tilde{\alpha}_{t+h} \\
\tilde{\sigma}_{t+h} \\
\tilde{\kappa}_{t+h} \\
\tilde{A}_{x, t+h} \\
\tilde{A}_{y, t+h} \\
\tilde{\omega}_{t+h}
\end{array}\right)=\left(\begin{array}{c}
\hat{c}_{1} \\
\hat{c}_{2} \\
\hat{c}_{3} \\
\hat{c}_{4} \\
\hat{c}_{5} \\
\hat{c}_{6}
\end{array}\right)+\left(\begin{array}{cccccc}
\hat{\gamma}_{1} & 0 & 0 & 0 & 0 & 0 \\
0 & \hat{\gamma}_{2} & 0 & 0 & 0 & 0 \\
0 & 0 & \hat{\gamma}_{3} & 0 & 0 & 0 \\
0 & 0 & 0 & \hat{\gamma}_{4} & 0 & 0 \\
0 & 0 & 0 & 0 & \hat{\gamma}_{5} & 0 \\
0 & 0 & 0 & 0 & 0 & \hat{\gamma}_{6}
\end{array}\right) \times\left(\begin{array}{c}
\hat{\alpha}_{t+h-1} \\
\hat{\sigma}_{t+h-1} \\
\hat{\kappa}_{t+h-1} \\
\hat{A}_{x, t+h-1} \\
\hat{A}_{y, t+h-1} \\
\hat{\omega}_{t+h-1}
\end{array}\right)
$$

The predicted value of the yield to maturity is given by

$$
\begin{aligned}
& \tilde{R}\left(r_{t+h}, t+h, T_{j}\right)=\left(z_{1 j, t+h}+1\right) r_{t+h}+z_{1 j, t+h} \tilde{\alpha}_{t+h}+z_{2 j, t+h} \tilde{\sigma}_{t+h}^{2} \\
+ & \operatorname{Re}\left[\frac { \tilde { A } _ { x , t + h } + i \tilde { A } _ { y , t + h } } { \tilde { \omega } _ { t + h } ( \tilde { \kappa } _ { t + h } + i \tilde { \omega } _ { t + h } ) } \left(e^{i \tilde{\omega}_{t+h}(t+h)}\left(\tilde{\omega}_{t+h} e^{-\tilde{\kappa}_{t+h}\left(T_{j}-(t+h)\right)}+i \tilde{\kappa}_{t+h}-\tilde{\omega}_{t+h}\right)\right.\right. \\
& \left.\left.-i \tilde{\kappa}_{t+h} e^{i \tilde{\omega}_{t+h} T_{j}}\right)\right] .
\end{aligned}
$$

Data for $z_{1 j, t+h}$ and $z_{2 j, t+h}$ are obtained from equations (12) and (13) using the predicted value of $\widetilde{\kappa}_{t+h}$.

i) From an upward-sloping to a flat yield curve: August 3, 2004, to August 2, 2005.

This first forecasting period is very interesting because of the significant changes in the TSIR over time. The TSIR is initially upward sloping with yields increasing with maturity and a spread between short-term and long-term rates of around 400 basis points. However, the gap between maturities drops to around 100 basis points by the end of the period.

Figure 3 shows the observed time-series yield for maturities from 1 month to 30 years. We see a significant change over time, with short-term rates displaying a noticeable increase of more than 200 basis points during the period. Medium-term yields converge to a common level of around $4 \%$ 
at the end of the period. Long-term rates stay roughly constant over the whole period, except for the 20-year and 30-year rates, which decrease by almost 100 basis points.

From December 1, 2005, forward, the 20-year rate exceeds the 30-year rate, producing a humped yield curve at its longer end. The lower right graph shows a gradual and significant flattening of the TSIR over the period. Hence, this first period can provide some insight as to how the models respond to changes in the level or shape of the yield curve.

Figure 4 shows 1 day ahead predictions from each model for 1 to 5 year maturities, as well as the corresponding forecasting errors, in absolute value. We can see that the three models forecast well at the beginning of the sample, when the TSIR has a clear upward shape. At the beginning of 2005, as well as in the last part of the sample, the Fourier model clearly predicts better than the Vasicek and Nelson-Siegel models, except at the 3-year maturity.

ii) Erratic short-term rates: August 2, 2006, to July 31, $200 \%$.

Figure 5 presents the observed yield time-series for each maturity between 1 month and 30 years for this second forecasting period. This period is more erratic, with several ups and downs in yield levels, especially at shorter maturities. The sizable fluctuations observed at some points for short-term rates are not repeated for the medium and long sections of the yield curve, that remain relatively flat over the whole period. In fact, the slope of the TSIR stays always between -100 and 100 basis points.

iii) Stable, low interest rates: June 19, 2015, to June 20, 2016.

Figure 2 shows a positive slope in the yield curve over the period. In addition, short-term rates are extremely low during the whole period so that even a slight deviation from the realized yield produces a huge relative forecast error. Figure 6 shows the observed time series yield for each maturity between 1 month and 30 years. Interest rates were quite stable over the period, displaying small fluctuations around their mean value. Short-term maturities remained particularly low, with 1 and 3 month yields staying in the neighborhood of zero during the first half of the sample. The TSIR slope was around 260 basis points in that first half. At that point, slightly higher short-term rates and lower long-term rates led to a significant reduction in the slope.

To formally test for statistically significant differences in forecasting per- 
formance between models, we use the Diebold-Mariano (DM) test (see Diebold and Mariano (1995)) with a quadratic loss function, $g\left(e_{i j}\right)=e_{i j}^{2}$; this is a standard assumption in forecast comparison. ${ }^{8}$ With this quadratic loss function, the model with the least squared forecast error is the potentially best forecasting model. Hence we apply a one-sided test. We take our Fourier model as model 1 in the test while the Nelson-Siegel and Vasicek models are taken alternately as model 2. Hence, when the Fourier model has lower mean squared forecast error, the DM test statistic will be negative, and it will be positive otherwise. Table 3 presents the sum of the squared forecast errors for each model, maturity, and forecasting horizon. Table 4 summarizes the results of the DM test that compares forecasts from our Fourier model with those of the Nelson-Siegel and Vasicek models. In the table we indicate whether differences across models, positive or negative, are statistically significant, so indicating better forecasting performance.

Forecast variance and, hence, the value of $f_{d}(0)$, increases with the forecast horizon. This explains the reduction in the value of the test statistic when we increase the forecast horizon in the three periods. Looking at maturities, the Fourier model yields significantly better 1 day ahead forecasts than the Nelson-Siegel model in 17 cases, versus 11 cases in which the Nelson-Siegel model generates statistically better forecasts. At this horizon, differences are generally significant even at the 0.001 significance level. At the 5 day ahead horizon, our Fourier model yields significantly better forecasts than NelsonSiegel in 14 cases, versus 8 cases in which the Nelson-Siegel model produces statistically better forecasts.

The generalization embedded in our Fourier model leads to statistically better 1 day ahead forecasts than the Vasicek model at 5\% significance in 26 cases, compared with 3 cases in which the Vasicek model generates better forecasts. At the 5 day horizon, the Fourier model beats the Vasicek model in 21 cases, with Vasicek being a better forecasting model in 3 cases.

At the 21 day horizon, there are just a few instances in which forecasts are significantly different. Our Fourier model beats the Nelson-Siegel model in 4 cases at $5 \%$ significance, with the opposite never being the case. It also yields significantly better forecasts than the Vasicek model in 4 cases, with Vasicek producing better forecasts in 1 case.

\footnotetext{
${ }^{8}$ An absolute value error function would yield the same results as the quadratic loss function, but the results of the test might change for other alternative loss functions.
} 
Considering the three forecasting periods we can conclude that the Fourier model outperforms both the Vasicek and the Nelson-Siegel models. These two models often produce much larger forecast errors than the Fourier model. On the other hand, when the Fourier model is beaten by either the Vasicek or the Nelson-Siegel model, the difference in performance is generally small. This is an additional important consideration in favor of preferring the Fourier model to the other alternatives when forecasting TSIR.

\section{Conclusions}

Many models have been proposed for describing the term structure of interest rates (TSIR), the relationship between interest rates and maturity at a given point in time. Characterizing the relationship between long-term and short-term rates, TSIR models are needed for the design and implementation of monetary policy, since the monetary authority can directly influence only the very short-term interest rate. Stochastic (Vasicek (1977), Cox et al. (1985), among others) and non-stochastic TSIR models currently used at central banks (Nelson and Siegel (1987), Svensson (1994)) are very flexible in their assumptions regarding the functional dependency between interest rates and their maturities.

We have introduced an alternative one-factor model for the TSIR that assumes the instantaneous interest rate to follow a stochastic process that reverts to a mean level displaying cyclical fluctuations. Such fluctuations seek to capture the dependence of the interest rates on the business cycle as the monetary authority tends to lower interest rates in recessions and raise them in economic expansions. Our specification extends the Vasicek (1977) model while preserving its analytical tractability. It also allows considerable flexibility, as it is able to capture a variety of different possible shapes for the TSIR.

In this framework, we have analyzed the empirical (in-sample and outof-sample) performance of a simple version of our Fourier model taking the Vasicek (1977) and Nelson and Siegel (1987) models as benchmarks, by forecasting the daily term structure over three periods that present specific peculiarities. Our findings show that our model provides better and more reliable in-sample and out-of-sample estimation of the yield curve, outperforming both competitors and providing more accurate forecasts. Furthermore, when the alternatives outperform our model, the difference in performance is relatively small. These results suggest that our Fourier model can provide a 
simple and powerful tool for empirical analysis and for potential applications for pricing and risk management. 


\section{References}

[1] Andersen, L.B. and V. Piterbarg (2010). Interest Rate Modeling, Atlantic Financial Press.

[2] Brigo, D. and F. Mercurio (2006). Interest Rate Models - Theory and Practice, Springer-Verlag Berlin Heidelberg.

[3] Cochrane, J.H. and M. Piazzesi (2005). Bond Risk Premia. American Economic Review, 95, 1, 138-160.

[4] Cox, J.C., J.E. Ingersoll, and S.A. Ross (1985). A Theory of the Term Structure of Interest Rates. Econometrica, 53, 2, 385-408.

[5] Diebold, F.X. and C. Li (2006). Forecasting the Term Structure of Government Bond Yields. Journal of Econometrics, 130, 337-364.

[6] Diebold, F.X. and R.S. Mariano (1995). Comparing Predictive Accuracy. Journal of Business and Economic Statistics, 13: 253-63.

[7] Diebold, F.X., Rudebusch, G.D., and S.B. Aruoba (2006). The Macroeconomy and the Yield Curve: A Dynamic Latent Factor Approach. Journal of Econometrics, 131, 309-338.

[8] Duffie, D. and R. Kan (1996). A Yield-Factor Model of Interest Rates. Mathematical Finance, 6, 4, 379-406.

[9] Evans, C.L. and D. Marshall (2007). Economic Determinants of the Nominal Treasury Yield Curve. Journal of Monetary Economics, 54, 1986-2003.

[10] Fama, E. and R. Bliss (1987). The Information in Long-Maturity Forward Rates. American Economic Review, 77, 680-692.

[11] Filipović, D. (2009). Term Structure Models - A Graduate Course, Springer-Verlag Berlin Heidelberg.

[12] Gourieroux, C. and A. Monfort (2013). Linear-Price Term Structure Models. Journal of Empirical Finance, 24, 24-41.

[13] Heath, D., R. Jarrow, and A. Morton (1992). Bond Pricing and the Term Structure of Interest Rates: A New Methodology for Contingent Claims Valuation. Econometrica, 60, 77-105. 
[14] Hull, J. and A. White (1990). Pricing Interest-Rate-Derivative Securities. Review of Financial Studies, 3, 4, 573-592.

[15] Hull, J. and A. White (1993). One-Factor Interest-Rate Models and the Valuation of Interest-Rate Derivative Securities. Journal of Financial and Quantitative Analysis, 28, 2, 235-254.

[16] Hull, J. and A. White (1994). Branching Out. Risk, 7, 34-37.

[17] James, J. and N. Webber (2001). Interest Rate Modelling: Financial Engineering, John Wiley \& Sons, Ltd, England.

[18] Mallick, S., M.S. Mohanty, and F. Zampolli (2017). Market Volatility, Monetary Policy and the Term Premium. BIS Working Papers No. 606, available at http://www.bis.org/publ/work606.pdf.

[19] Moreno, M. and F. Platania (2015). A Cyclical Square-Root Model for the Term Structure of Interest Rates. European Journal of Operational Research, 241, 1, 109-121.

[20] Moreno, M., A. Novales, and F. Platania (2016). A Term Structure Representation with Cyclical Mean Reversion. Working paper (available upon request).

[21] Munk, C. (2015). Fixed Income Modelling, Oxford University Press, United Kingdom.

[22] Nawalkha, S.K., N.A. Believa, and G.M. Soto (2007) Dynamic Term Structure Modeling. John Wiley \& Sons, Ltd, Hoboken.

[23] Nelson, C.R. and A.F. Siegel (1987). Parsimonious Modeling of Yield Curves. Journal of Business, 60, 4, 473-489.

[24] Paccagnini, A. (2016). The Macroeconomic Determinants of the US Term Structure during the Great Moderation. Economic Modelling, 52, A, 216-225

[25] Realdon, M. (2016). Tests of Non Linear Gaussian Term Structure Models. Journal of International Financial Markets, Institutions and Money, 44, 128-147. 
[26] Renne, J.P. (2016). A Tractable Interest Rate Model with Explicit Monetary Policy Rates. European Journal of Operational Research, 251, 3, 873-887.

[27] Svensson, L.E.O. (1994). Estimating and Interpreting Forward Interest Rates: Sweden 1992 - 1994. IMF Working Paper 94/114

[28] Tillmann, P. (2007). Inflation Regimes in the US Term Structure of Interest Rates. Economic Modelling, 24, 2, 203-223

[29] Vasicek, O. (1977). An Equilibrium Characterization of the Term Structure. Journal of Financial Economics, 5, 2, 177-188. 


\section{Appendix of Tables}

Table 1: Parameter estimates. In-sample estimation

\begin{tabular}{|lccc|}
\hline \hline Parameter & Fourier & Vasicek & Nelson-Siegel \\
\hline \hline$\hat{\delta}_{1} \times 100$ & $4.949(0.003)$ & $4.983(0.056)$ & - \\
$\hat{\beta}_{1} \times 100$ & - & - & $4.609(0.002)$ \\
$\hat{\delta}_{2} \times 100$ & $0.029(0.000)$ & $0.158(0.031)$ & - \\
$\hat{\beta}_{2} \times 100$ & - & - & $-3.295(0.002)$ \\
$\hat{\delta}_{3} \times 100$ & $0.063(0.005)$ & - & - \\
$\hat{\beta}_{3} \times 100$ & - & - & $-2.906(0.003)$ \\
$\hat{\delta}_{4} \times 100$ & $-0.354(0.006)$ & - & - \\
$\hat{\kappa}$ & $0.239(0.000)$ & $0.349(0.003)$ & - \\
$\hat{\lambda}$ & - & - & $0.590(0.001)$ \\
$\hat{\omega}$ & $1.030(0.000)$ & - & - \\
\hline \hline$\sum_{i, t} \min \operatorname{SR}\left(\hat{\theta}_{i, t}\right)$ & 177 & 1118 & 229 \\
$\sum_{i, t}\left|\hat{u}_{i, t}\right|$ & 1892 & 5172 & 2184 \\
\hline
\end{tabular}

Note: This table presents the mean and standard deviation (in parenthesis) of the daily time series of estimated parameters over the whole sample period, July 31, 2001, to June 20, 2016 (3724 days). $\sum_{i, t} \min \operatorname{SR}\left(\hat{\theta}_{i, t}\right)$ and $\sum_{i, t}\left|\hat{u}_{i, t}\right|$ denote, respectively, the sum of squared errors and the sum of their absolute values for the whole period and every maturity. 
Table 2: Goodness of fit statistics over the whole sample period, July 31, 2001, to June 20, 2016 (3724 days).

\begin{tabular}{|l|cc|cc|cc|}
\hline \hline \multirow{2}{*}{ Maturity } & \multicolumn{2}{|c|}{ Fourier } & \multicolumn{2}{|c|}{ Vasicek } & \multicolumn{2}{c|}{ Nelson-Siegel } \\
& $\sum_{t} \hat{u}_{t}^{2}$ & $\sum_{t}\left|\hat{u}_{t}\right|$ & $\sum_{t} \hat{u}_{t}^{2}$ & $\sum_{t}\left|\hat{u}_{t}\right|$ & $\sum_{t} \hat{u}_{t}^{2}$ & $\sum_{t}\left|\hat{u}_{t}\right|$ \\
\hline \hline 3 months & 21 & 209 & 53 & 363 & $\mathbf{7}$ & $\mathbf{1 0 1}$ \\
\hline 6 months & $\mathbf{2 6}$ & 237 & 132 & 584 & 32 & $\mathbf{2 3 6}$ \\
\hline 1 year & $\mathbf{9}$ & $\mathbf{1 4 1}$ & 178 & 692 & 22 & 210 \\
\hline 2 years & 12 & 149 & 207 & 714 & $\mathbf{8}$ & $\mathbf{1 3 1}$ \\
\hline 3 years & 16 & 197 & 183 & 643 & $\mathbf{1 0}$ & $\mathbf{1 4 0}$ \\
\hline 5 years & 15 & 178 & 67 & 409 & $\mathbf{1 2}$ & $\mathbf{1 6 9}$ \\
\hline 7 years & 13 & 172 & 71 & 445 & $\mathbf{1 2}$ & $\mathbf{1 6 4}$ \\
\hline 10 years & $\mathbf{1 1}$ & $\mathbf{1 3 8}$ & 74 & 425 & 19 & 225 \\
\hline 20 years & $\mathbf{3 4}$ & $\mathbf{2 7 3}$ & 86 & 490 & 48 & 352 \\
\hline 30 years & $\mathbf{2 1}$ & $\mathbf{1 9 7}$ & 67 & 408 & 22 & 211 \\
\hline
\end{tabular}

Note: This table presents the goodness of fit statistics over the whole sample for each model and maturity. Figures in boldface denote the lowest numerical value across the three models. 
Table 3: Out-of-sample 1, 5, and 21 day ahead forecasting errors.

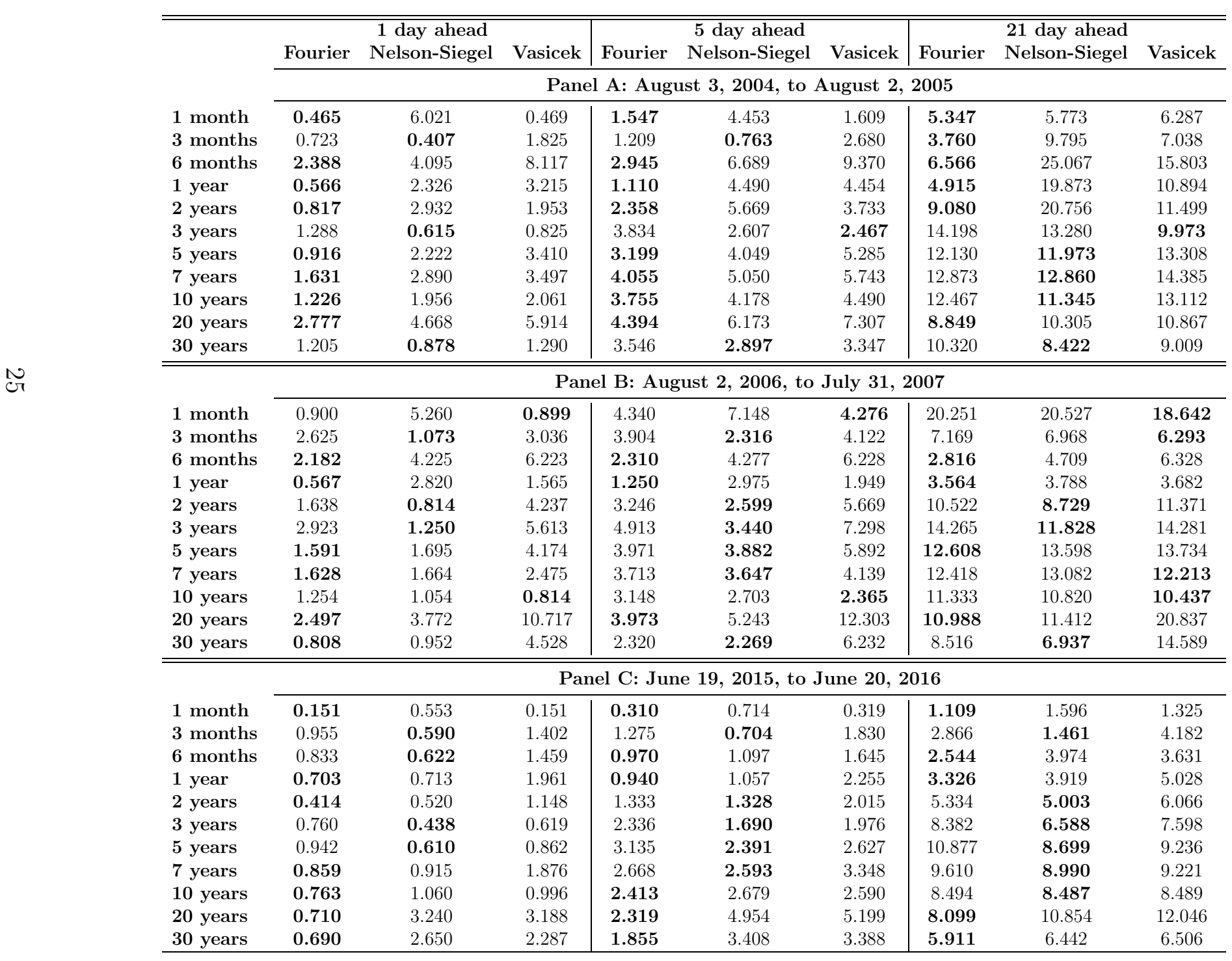

Note: The table displays the sums of 1,5 , and 21 day ahead squared forecasting errors for each model and maturity.

Figures in bold face denote the lowest value of the sum of squared errors across models. 
Table 4: Diebold-Mariano test.

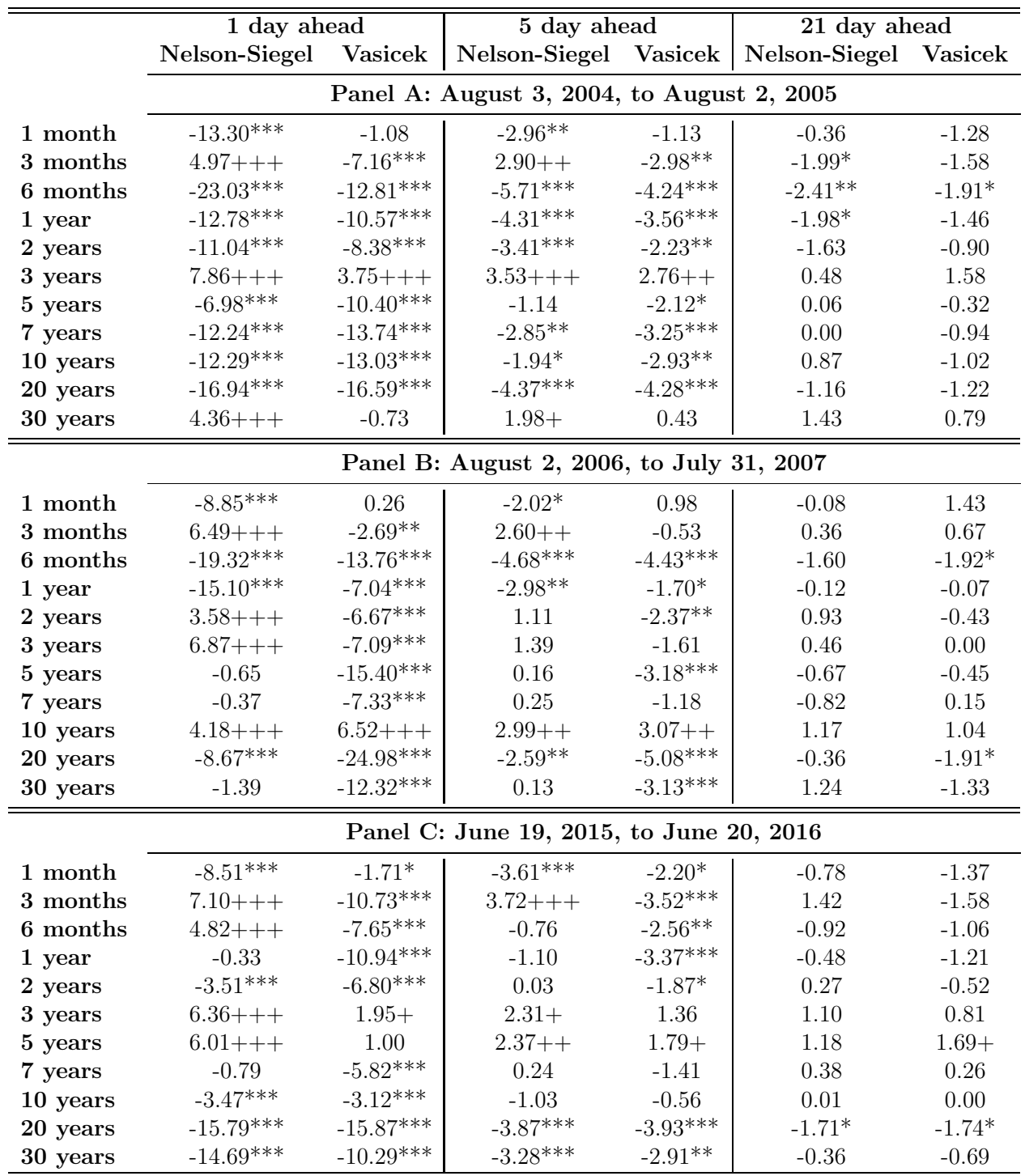

Note: This table displays the values of the Diebold-Mariano statistic to test for forecasting differences between the Fourier model, Nelson-Siegel model (left column), and Vasicek model (right column). Stars (plus symbols) denote periods where the Fourier model has statistically better (worse) forecasting performance: $*(+)$ denotes a p-value between 0.01 to $0.05,{ }^{* *}(++)$ a p-value between 0.001 to $0.01, * * *(+++)$ a p-value less than 0.001 


\section{Appendix of Figures}

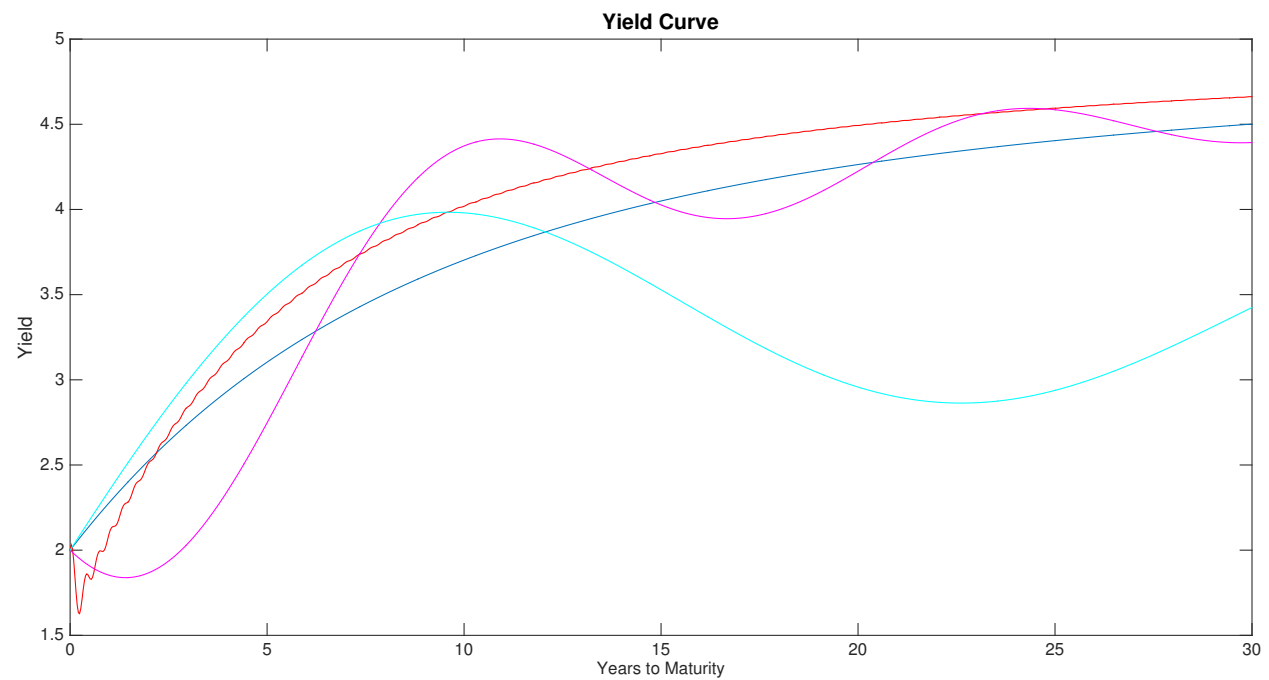

Figure 1: Term structure of interest rates for some parameter vectors in the Vasicek and Fourier models. In all the cases, $r_{0}=0.02$. For the Vasicek model (blue line) we consider $\alpha=0.05, \sigma=0.002$, and $\kappa=0.2$. For the Fourier model, we consider three alternatives: a) Red line: $\alpha=0.05, \sigma=0.0011, \kappa=0.3397, \omega=20, n=5, A_{1, x}=0.1758, A_{1, y}=0.0402, A_{2, x}=$ $-0.3011, A_{2, y}=0.0172, A_{3, x}=0.0498, A_{3, y}=-0.1215, A_{4, x}=0.0798, A_{4, y}=0.1618, A_{5, x}=0.0894, A_{5, y}=0.0655$. b) Green line: $\alpha=0.07, \sigma=0.0005, \kappa=0.018, \omega=0.48, n=2, A_{1, x}=-1.8, A_{1, y}=1, A_{2, x}=1.5, A_{2, y}=-1.5$. c) Violet line: $\alpha=0.08, \sigma=0.0002, \kappa=0.02, \omega=0.25, n=1, A_{1, x}=0.3, A_{1, y}=0.03$. 

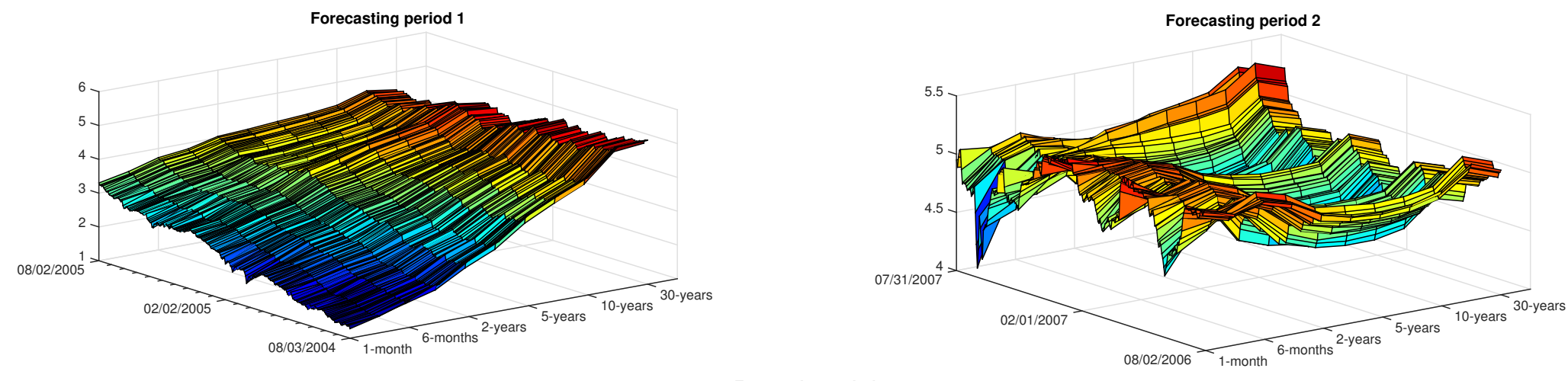

$\stackrel{\infty}{\infty}$

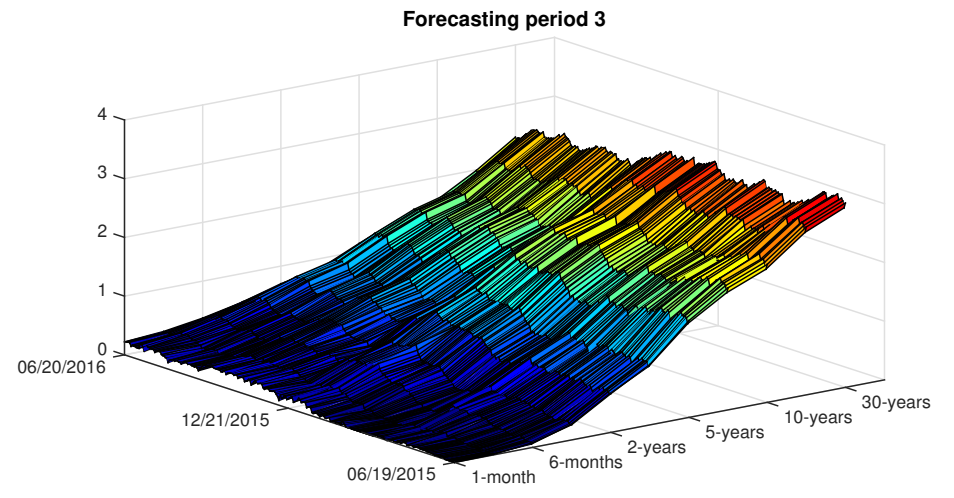

Figure 2: Observed term structure of interest rates for the three forecasting periods. These periods range, respectively, from August 3, 2004, to August 2, 2005, from August 2, 2006, to July 31, 2007, and from June 19, 2015, to June 20, 2016. 

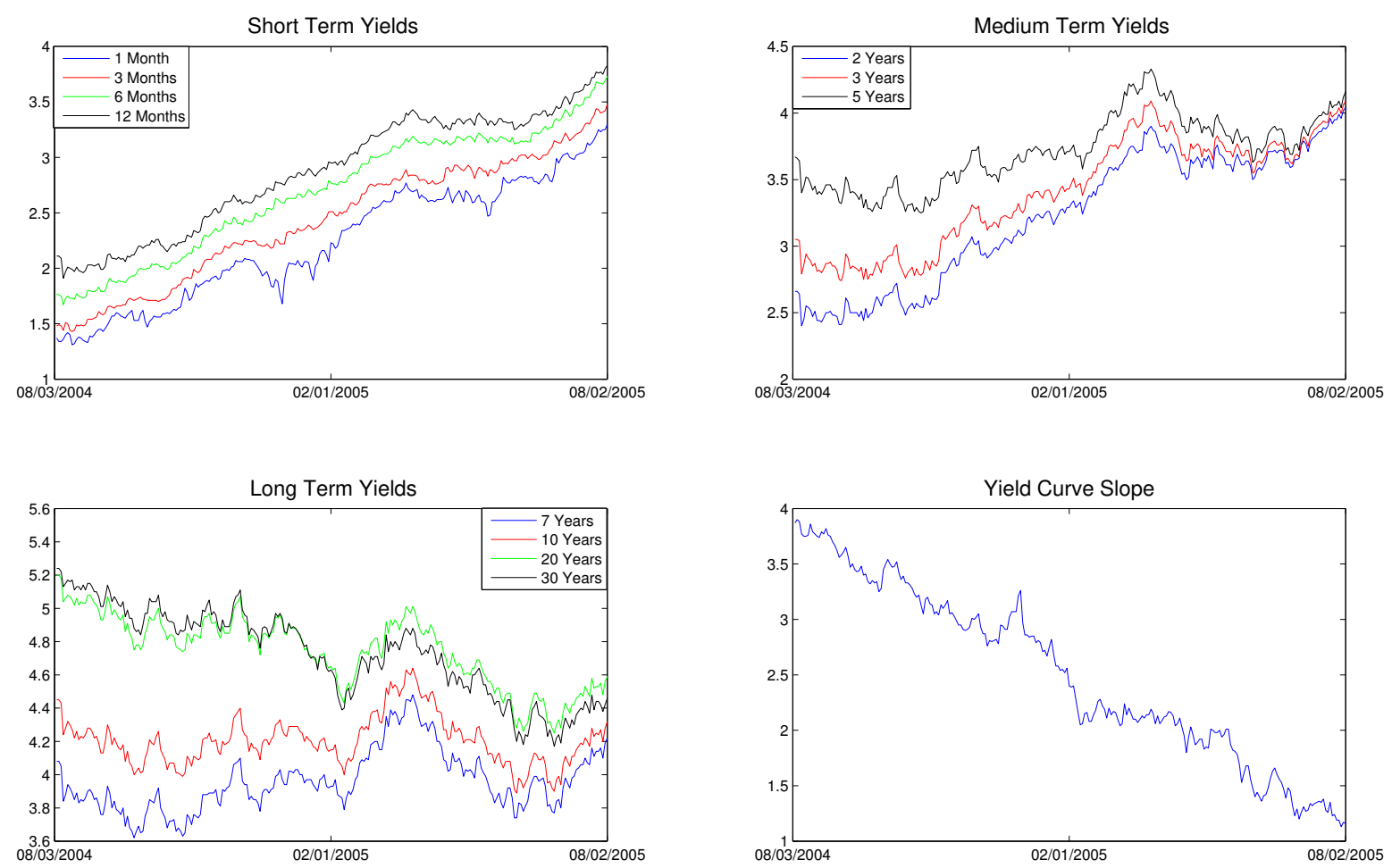

Figure 3: Yields and term structure slope over the first forecasting period, from August 3, 2004, to August 2, 2005. 

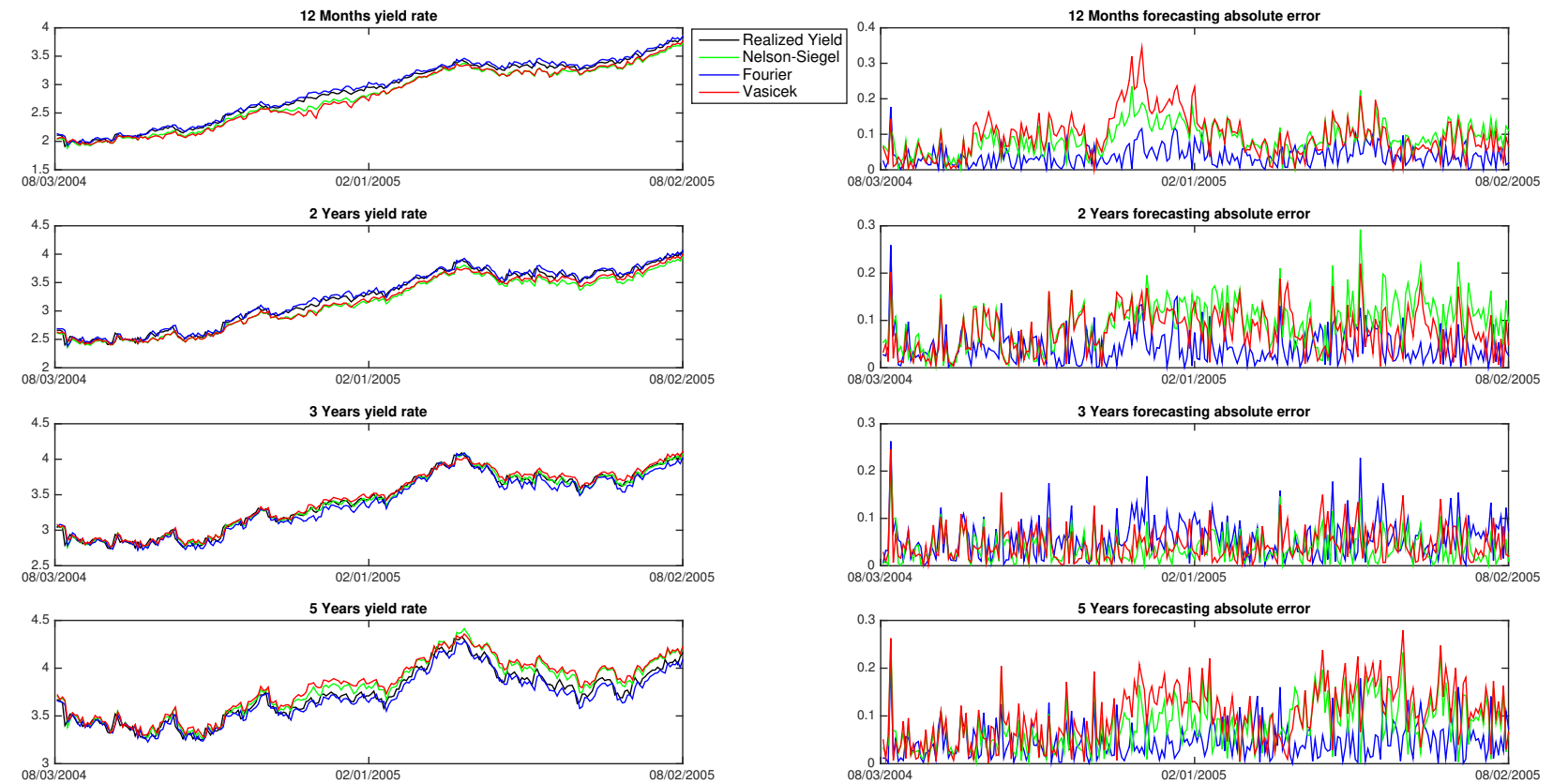

Figure 4: 1 day ahead predictions from each model for 1 to 5 year maturities and the associated forecasting errors (in absolute value) for the first forecasting period, from August 3, 2004, to August 2, 2005. 

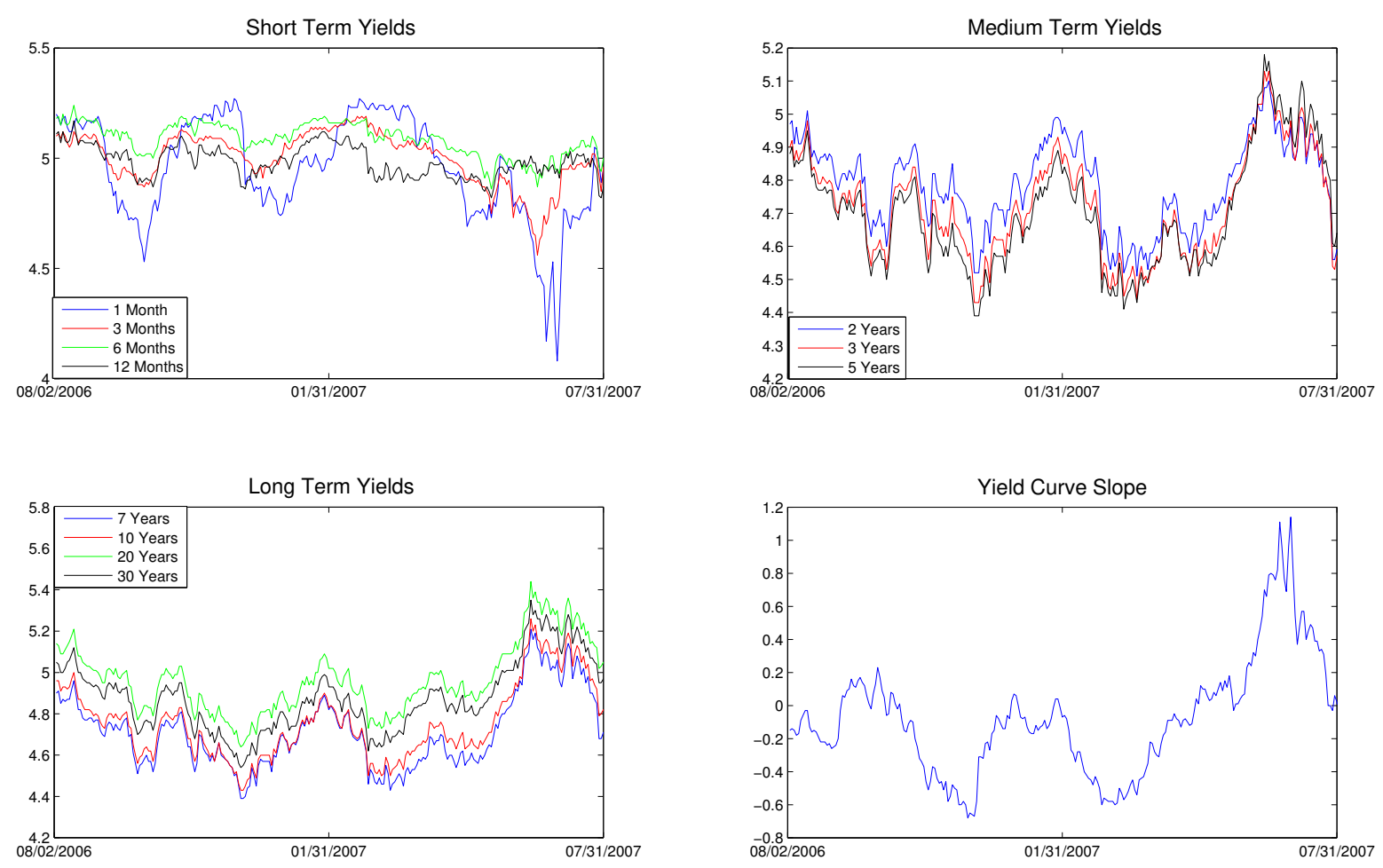

Figure 5: Yields and term structure slope over the second forecasting period, from August 2, 2006, to July 31, 2007. 

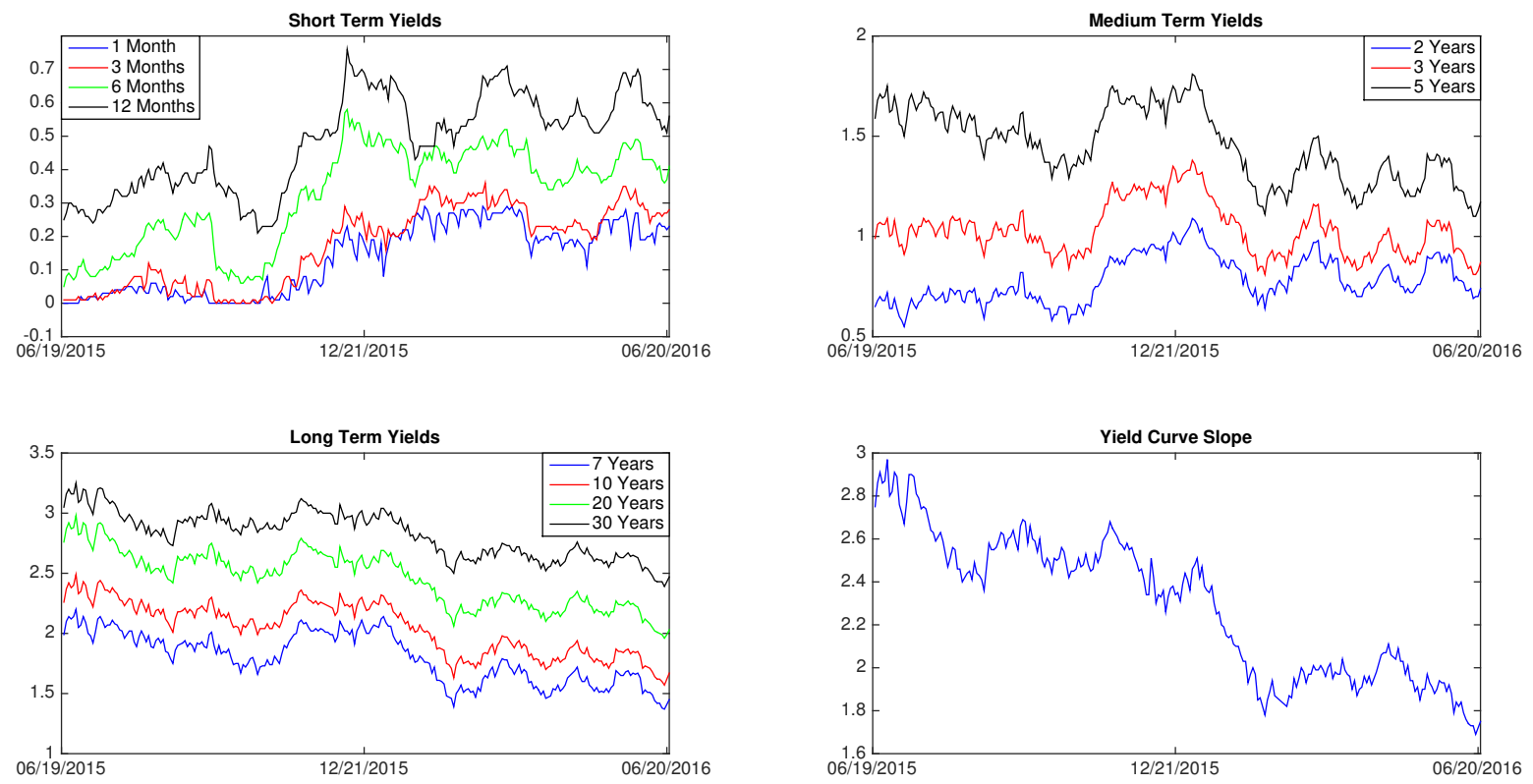

Figure 6: Yields and term structure slope over the third forecasting period, from June 19, 2015, to June 20, 2016. 